\title{
Performance Analysis of Slotted Fiber-Optic Code- Division Multiple-Access (CDMA) Packet Networks
}

\author{
Cherng-Shung Hsu, Member, IEEE, and Victor O. K. Li, Fellow, IEEE
}

\begin{abstract}
This paper examines code-division multiple-access (CDMA) techniques used in slotted fiber-optic packet networks. Since the inherent properties and signal processing of the conventional communication channels are different from those of the fiber-optic channels, new code sequences must be constructed for fiber-optic applications. The goal of our research is to analyze the performance of fiber-optic CDMA packet networks using code sequences with given orthogonality properties.
\end{abstract}

Index Terms - Code-division multiple access, fiber-optic networks, packet networks, performance analysis.

\section{INTRODUCTION}

$\mathbf{T}$ HE evolution of fiber-optic technology makes it possible to introduce many new services which cannot be supported by today's copper cables. This is because optical fibers have a much larger bandwidth-distance product than copper cables. To take full advantage of the wide bandwidth available with optical fibers, it is necessary to process the signals optically. Spread-spectrum signaling has been proposed to achieve multiuser capability in fiber-optic CDMA networks. Due to the interference rejection capability of CDMA networks, multiple successful transmissions can be achieved. These access schemes tend to be more efficient for large user population, bursty traffic, and short access delays.

\section{A. Fiber-Optic Code Sequences}

Fiber-optic signal processing today uses incoherent detection, which is based on power measurements. Typically, fiberoptic signal processors and fiber-optic systems are modeled as positive systems [1], [2]. Besides, a correlator is used to process the signals. However, the processing is "optical." Since the measured power is always positive, it is impossible to manipulate the signals so they add to zero. Therefore, conventional code sequences [3], [4] designed for +1 and -1 signals cannot be applied to fiber-optic systems. Thus, a set of new code sequences [5]-[8] must be constructed for fiber-optic applications.

Paper approved by I. Chlamtac, the Editor for Computer Networks of the IEEE Communications Society. Manuscript received May 15, 1992; revised January 15, 1995 and July 15, 1996. This paper is part of a dissertation submitted by C.-S. Hsu to the University of Southern California, Los Angeles, in partial fulfillment of the requirements for the Ph.D. degree. The work of V. O. K. Li was supported in part by the NSF under Grant NCR-9318497. This paper was presented in part at the International Conference on Computer Communications and Networks, San Diego, CA, June 1992.

C.-S. Hsu is with the Chung-Shan Institute of Science and Technology, Lung-Tan, Taiwan, R.O.C.

V. O. K. Li is with the Department of Electrical Engineering, Communication Sciences Institute, University of Southern California, Los Angeles, CA 90089-2565 USA (e-mail: vli@irving.usc.edu).

Publisher Item Identifier S 0090-6778(97)05183-0.
Any new code sequences $x_{n}, y_{n}, n=0,1, \cdots, c-1$ should satisfy the following two conditions [8]-[10].

1) For any sequence $x_{n}$ in the set, the autocorrelation of $x_{n}$ is defined as

$$
R_{x x}(\tau) \equiv \sum_{n=0}^{c-1} x_{n} x_{n \ominus \tau}= \begin{cases}d, & \text { for } \tau=0 \\ \leq \lambda_{a}, & \text { for } 1 \leq \tau \leq c-1\end{cases}
$$

2) For each pair of sequences $x_{n}$ and $y_{n}$, the crosscorrelation of $x_{n}$ and $y_{n}$ is defined as

$$
R_{x y}(\tau) \equiv \sum_{n=0}^{c-1} x_{n} y_{n \ominus \tau} \leq \lambda_{c}, \quad \text { for } 0 \leq \tau \leq c-1
$$

where $\oplus$ is modulo $c$ addition, $c$ is the length of the code sequence, and $d$ is the weight of the code sequence.

Real orthogonality requires that $\lambda_{a}=\lambda_{c}=0$. But in the positive system, $\lambda_{a} \neq 0$ and $\lambda_{c} \neq 0$, and the minimum values are 1 . If a set of codes has minimum autocorrelation and cross correlation, then we say that these codes satisfy orthogonality in the "optical" sense. We will use the shorthand notation $(c, d, 1)$ to represent a code with length $c$, weight $d$, and $\lambda_{a}=\lambda_{c}=1$ [5].

\section{B. Transmitted Signal Format and Receiving Subsystem of Fiber-Optic CDMA System}

The format of transmitted signals in typical fiber-optic codedivision multiplier access (CDMA) communication networks is on-off keying (OOK) [11] which is shown in Fig. 1(b). In the OOK format, a " 1 " bit is transmitted as a pulse signature pattern and a " 0 " bit is transmitted as an empty bit (i.e., no pulse signature pattern is transmitted).

The receiving subsystem shown in Fig. 1(c) includes three main parts, namely, the optical correlator, the photodetector, and the threshold detector. Basically, the optical correlator just adds the power levels at some chip positions according to the designed signature pattern. The light intensity of the correlator output is detected by the photodetector. Then the photodetector output is sampled every $T_{b}$ seconds (i.e., one bit period). However, the timing information is derived from a synchronization subsystem which is not shown in Fig. 1(c). In our analysis, we will assume that this timing information can be obtained, and that the synchronization is perfect. Finally, the sampled signal is sent to a threshold detector. If the input level to the threshold detector is above the threshold $T h$, the recovered bit is decoded as " 1 "; otherwise, it is decoded as "0." Due to the positive property of fiber-optic systems, if bit " 1 " is transmitted, then it will always be correctly decoded, and bit errors may occur only when the " 0 " bit is transmitted. 


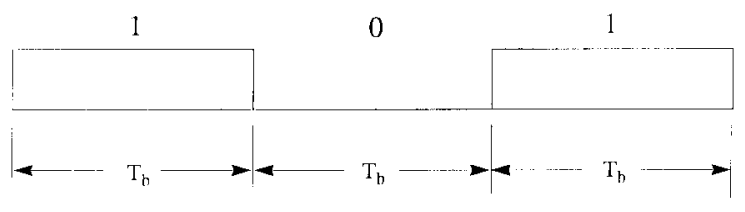

(a)

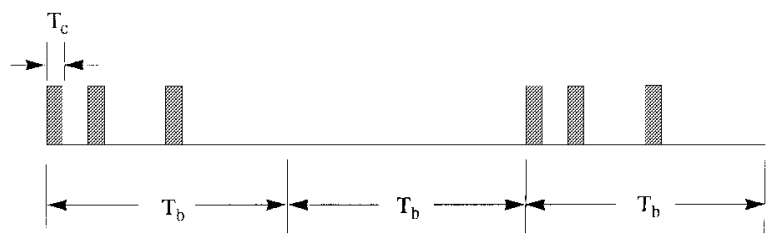

(b)

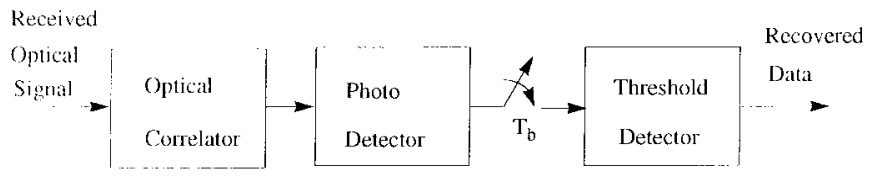

(c)

Fig. 1. Signal format and receiving subsystem for fiber-optic CDMA system.

There is very limited research on the performance of fiberoptic packet CDMA networks [12], [13]. In [12], Gelman and Schilling assume that the interference due to different users are uncorrelated, so that the bits in the packet are affected by the interference independently. They derive the throughput for a hybrid time-wavelength CDMA network. In [13], Hui proposes multiple symbol digital modulation for multifiber optical CDMA networks, and derives the theoretical capacity and the probability of false detection. Most of the research concentrates on the probability of bit error in fiberoptic CDMA networks [8]-[10], and it is assumed that the communication links are being used to transmit messages continuously (or for a very long period of time).

The rest of this paper is organized as follows. In Section II, we present the model, and then analyze some properties of fiber-optic code sequences relevant to our further analysis. In Section III, the performance analysis of a centralized slotted time hop/on-off keying (TH/OOK) and hybrid frequency hop/time hop/OOK (FH/TH/OOK) fiber-optic CDMA packet network is presented. We consider the throughput and delay of the network for different chip rates and probabilities of retransmission by taking the capture effect into consideration. In Section IV, we consider the distributed slotted fiber-optic CDMA packet network. We assume that a transmitter can always find an idle receiver waiting to receive this packet (i.e., nonpaired off) [14]. In this situation, the number of successful transmissions can be greater than one in a time slot. In the above two sections, we also evaluate the performance improvement by adding forward error-correcting (FEC) code and an optical hard limiter. We conclude in Section V.

\section{System Assumptions AND PROPERTIES OF SignAtures}

\section{A. System Assumptions}

The goal of our research is to analyze the performance of CDMA packet networks using code sequences with given orthogonality properties. There are several code sequences designed for use in fiber-optic CDMA [5]-[10]. We only consider $(c, d, 1)$ code sequences. The orthogonality properties of these code sequences allow us to extract useful information such as vulnerable positions and interference sets which are relevant to our analysis.

Since each bit of a packet is encoded by a specific signature pattern [we call it the time hopping (TH) pattern in the rest of the paper] which is only one bit long, the interferences due to other simultaneous transmissions are highly correlated. This means that the probabilities of bit errors in a packet are also highly correlated.

We consider both a centralized topology, in which all nodes transmit to a central node, and a fully connected distributed topology, in which a transmission by any node is heard by all nodes in the network.

In the analysis, we make the following assumptions.

1) Finite population assumption. The number of nodes $M$ in the network is finite.

2) Each packet consists of $N$ bits and takes $T$ seconds to transmit. Each bit is divided into $c$ chips (length of signature pattern), and the number of pulses (weight of signature pattern) in each bit is $d$.

3) Each node transmits with the same code.

4) Each node transmits with the same intensity. Without loss of generality, assume that this intensity is unity.

5) A transmission is received with equal light power by all other nodes. Since the loss of the optical fiber is very small, this assumption holds for high-speed local-area network applications. Besides, in centralized networks, all in-bound signals can be received with equal light power at the central station by using the in-bound power control scheme.

6) An idle receiver will capture the first packet addressed to it. The capture of this packet is successful only if any other packets are received at least one chip behind the first packet, and any other transmissions during this reception are treated as interference.

7) The analysis presented does not account for thermal noise. Instead, we are primarily concerned with multipleaccess interference due to other simultaneous transmissions.

8) A separate and error-free channel is used for acknowledgment. Since the acknowledgment is very short (a few bits), we can implement a very powerful forward error-correcting code to achieve error-free transmission with minimal additional bandwidth.

9) The timing information needed for synchronization is perfect.

10) For simplicity of analysis, the network is assumed to be chip-synchronized, namely, the time axis is divided into chips, and each packet can only be initiated at chip boundaries. With this assumption, our results will be pessimistic [9], [10].

11) Each packet consists of one codeword when errorcorrecting code is used.

\section{B. Vulnerable Positions and Interference Sets of TH Signatures}

Each receiver is assigned a code sequence, and each transmitter will use this signature pattern to encode their information bits. The encoding and decoding processes are described 


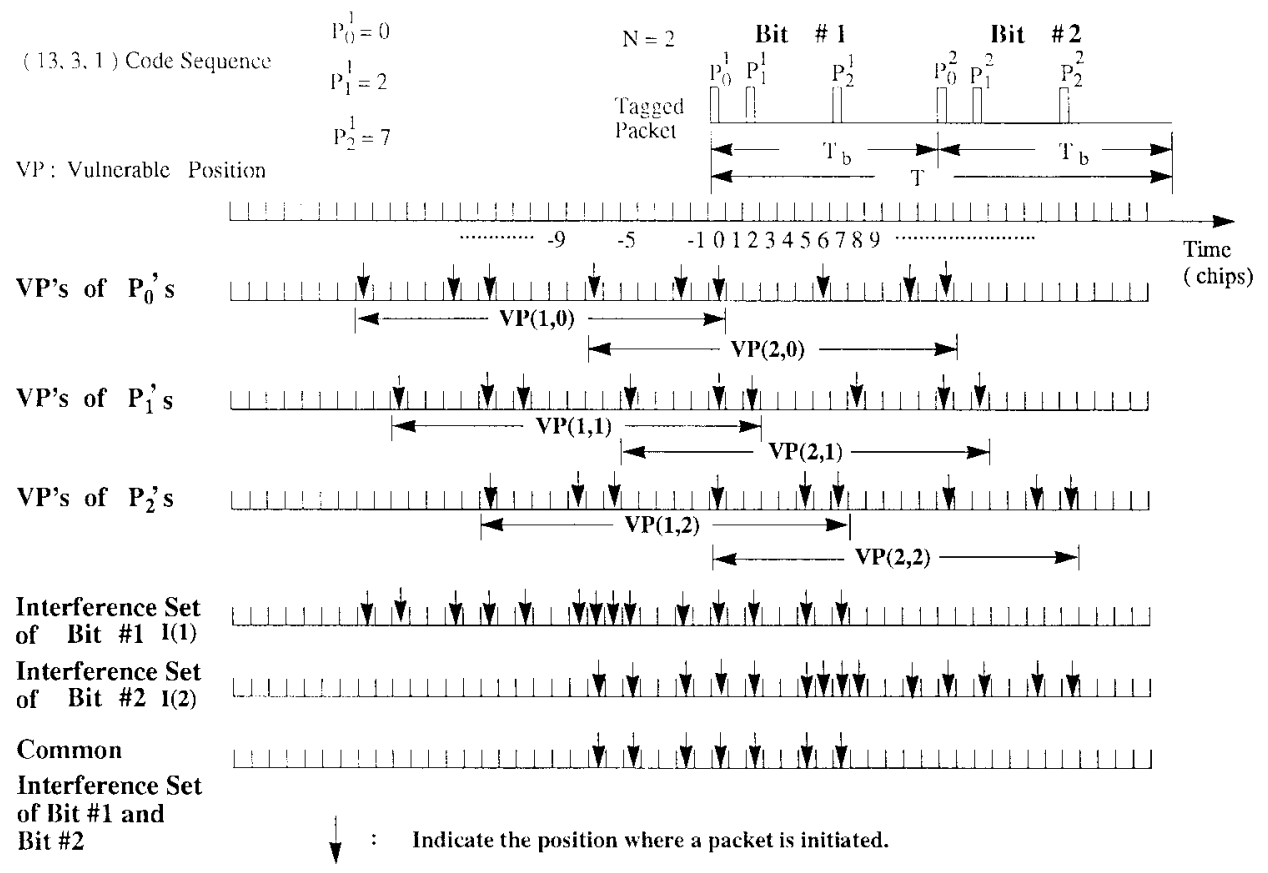

Fig. 2. Vulnerable positions and interference sets of $(13,3,1)$ code sequence.

in Section I-B. In this section, we want to characterize the interference between simultaneous transmissions which is relevant to the following performance analysis. We will find the interference sets which contribute interferences to a "tagged" packet. Since the signature pattern is repeated in every bit, we only have to concentrate on one tagged bit $\alpha$ of the tagged packet.

Let $P_{0}^{\alpha}, P_{1}^{\alpha}, \cdots, P_{d-1}^{\alpha}$ be the positions occupied by $d$ pulses in the tagged bit $\alpha$. These $d$ positions are selected according to the designed signature pattern. Now, consider pulse $P_{i}^{\alpha}$ of tagged bit $\alpha$. If any packet is initiated at positions

$$
\begin{aligned}
\Delta_{i j k}^{\alpha}=\left(P_{i}^{\alpha}-P_{j}^{\alpha}\right)-k \cdot c \\
\quad j=0, \cdots, d-1 ; \quad k=0, \cdots, N-1
\end{aligned}
$$

then it will interfere with the tagged pulse $P_{i}^{\alpha}$. These positions are defined as the vulnerable positions of pulse $P_{i}^{\alpha}$ of bit $\alpha$, and are denoted by $\operatorname{VP}(\alpha, i), i=0,1, \cdots, d-1$, i.e.,

$$
V P(\alpha, i) \equiv\left\{\Delta_{i j k}^{\alpha} \mid \begin{array}{l}
\Delta_{i j k}^{\alpha}=\left(P_{i}^{\alpha}-P_{j}^{\alpha}\right)-k \cdot c \\
j=0,1, \cdots, d-1, \\
k=0,1, \cdots, N-1
\end{array}\right\} .
$$

For each pulse, there are $N \cdot d$ vulnerable positions associated with it, i.e., $|V P(\alpha, i)|=N \cdot d$. If a packet is initiated at chip positions $P_{0}^{\alpha}-k \cdot c$, where $k=0,1, \cdots, N-1$, then it will interfere with all pulses of the tagged bit $\alpha$ at the same time. Thus, chip positions $P_{0}^{\alpha}-k \cdot c$, where $k=0,1, \cdots, N-1$ are common vulnerable positions for all $d$ pulses of tagged bit $\alpha$. The interference set of bit $\alpha$ is denoted by $I(\alpha)$ which is composed of vulnerable positions of all pulses of bit $\alpha$, namely,

$$
I(\alpha) \equiv\{V P(\alpha, i): i=0,1, \cdots, d-1\}
$$

There is a total of $N[1+d(d-1)]$ chip positions which are vulnerable positions of the pulses of tagged bit $\alpha$, i.e., $|I(\alpha)|=N[1+d(d-1)]$.

Example: Consider the $(13,3,1)$ code as shown in Fig. 2 where $c=13, d=3$, and $N=2$. We have $P_{0}^{1}=0, P_{1}^{1}=2$, $P_{2}^{1}=7,|V P(\alpha, i)|=N \cdot d=6,|I(\alpha)|=N[1+d(d-1)]=$ 14.

With these vulnerable positions and interference sets information, we can proceed to analyze fiber-optic CDMA packet networks in the following section.

\section{Centralized Slotted Fiber-Optic CDMA PACKET NETWORKS}

In a centralized network, we assume that there are $M$ users which communicate with a central station. This is called in-bound communication. The communication from the central station to users is called out-bound communication. Communication between users must go through the central station. We only consider the performance for the in-bound case.

\section{A. TH/OOK CDMA Packet Networks}

First, we analyze the TH/OOK scheme described in Section I-B.

1) Delay Capture Effect: In a CDMA network, the receiver can successfully receive the first of several overlapping packets even though each packet uses the same code sequence, provided that the first packet arrives at least a certain time $\left(T_{c}\right)$ before the other packets, and the later arriving packets do not have sufficient signal strength to overwhelm the interference rejection capability of the receiver. This capability gives rise to the delay capture property [3], [15], [16]. Because of the use of a spread-spectrum waveform, the performance characteristics 
of the system are quite different from those of the classical (nonspread-spectrum) ALOHA [17].

For a slotted system, each user with a packet may send it at the beginning of the slot. Hence, contending packets in a given slot arrive at the central station at time instants dependent on the distance to the central station. This leads to discrimination against users who are farther away from the central station due to the delay capture effect. This may be eliminated by using a time-of-arrival randomization procedure proposed by Davis and Gronemeyer [15]. This randomization procedure results in a uniform distribution of arrival times over the interval $\left(T_{g}, T_{g}+T_{u}\right)$, and eliminates discrimination against the more distant users. $T_{g}$ is a guard time, and $T_{u}$ can be chosen by the system. The capture probability of the first packet is given by [15]

$$
\begin{aligned}
P^{c}(k) & =\text { Prob }\left\{\begin{array}{l}
\text { the second packet arrives at } \\
\text { the central station at least } T_{c} \\
\text { seconds behind the first packet } \\
\text { given } k \text { packets arrive at the } \\
\text { central station in the same } \\
\text { time slot }
\end{array}\right\} \\
& = \begin{cases}1, & \text { if } k=1 \\
\left(1-\frac{T_{c}}{T_{u}}\right)^{k}, & \text { if } k \geq 2 \\
0, & \text { otherwise }\end{cases}
\end{aligned}
$$

where $T_{c}$ is the capture time. In our case, $\left(T_{c} / T_{u}\right)=(1 / c)$.

2) System Model and Analysis: In this section, we use a model similar to that developed by Kleinrock [18], Davis [15], and Carleial [19]. A Markovian model is first formulated for a population of $M$ users. Each user can be in one of two states, i.e., the thinking or the blocked state. In the thinking state, a user generates and transmits a new packet in a time slot with probability $q_{0}$. A user with a packet which is not correctly received enters the blocked state. A blocked user cannot generate new packets. The unsuccessful packet is retransmitted in the next slot with probability $q_{r}$.

Let $N_{b}^{t}$ be a random variable, called channel backlog, which represents the number of blocked users at the end of slot $t$. $N_{b}^{t}$ is a Markov chain having stationary transition probabilities, and provides the state description of the system. The discrete state space consists of states zero- $M$. The one-step state transition probabilities for $N_{b}^{t}$ are $P_{i j}=\operatorname{Prob}\left\{N_{b}^{t+1}=\right.$ $\left.j \mid N_{b}^{t}=i\right\}, i, j=0,1, \cdots, M$, and are given in (1a), shown at the bottom of the page.
The corresponding mathematical expressions are

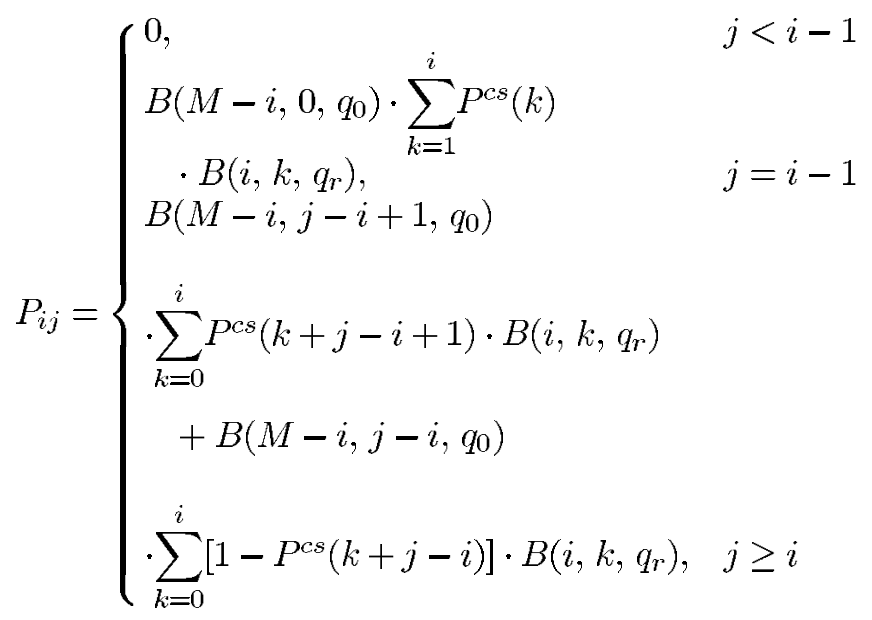

where

$$
B(n, k, p)=\left(\begin{array}{l}
n \\
k
\end{array}\right) p^{k}(1-p)^{n-k}
$$

is the probability mass function of the binomial distribution, and

$$
\left.\begin{array}{rl}
P^{c s}(k) & \equiv\left\{\begin{array}{l}
\text { one packet is received successfully } \\
\text { given } k \text { packets transmit in a } \\
\text { time slot simultaneously }
\end{array}\right\} \\
& =P^{c}(k) \cdot P^{s}(k)
\end{array}\right\}
$$

where the capture probability $P^{c}(k)$ is given by (1), and the conditional success probability is defined as

$$
\begin{aligned}
P^{s}(k) \equiv & \text { Prob }\left\{\begin{array}{l}
\text { the first arriving packet is received } \\
\text { successfully given that this packet is } \\
\text { captured and there are } k \text { packets which } \\
\text { transmit in a time slot simultaneously }
\end{array}\right\}
\end{aligned}
$$

which will be derived in the next section.

Since the Markov chain $N_{b}^{t}$ has a finite state space and is irreducible (assume $q_{0}, q_{r}>0$ ), a stationary probability distribution vector always exists [20]. The stationary probability distribution vector

$$
\boldsymbol{\Pi}=\left(\begin{array}{llll}
\pi_{0} & \pi_{1} & \cdots & \pi_{M}
\end{array}\right)
$$

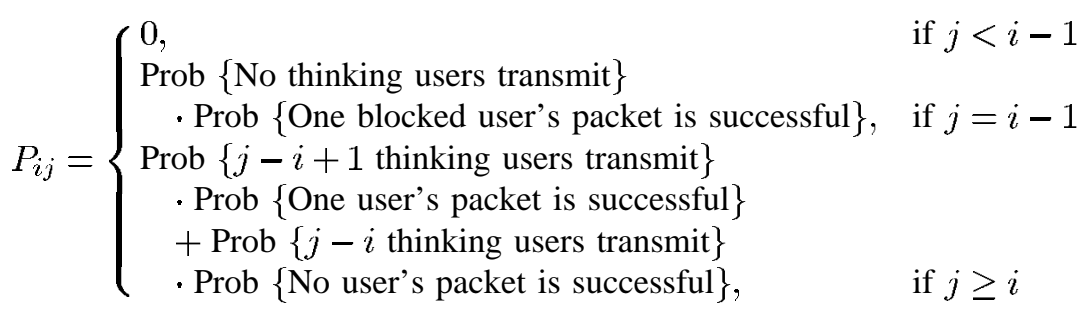


of $N_{b}^{t}$ can be computed by solving the following set of linear simultaneous equations

$$
\left\{\begin{array}{l}
\pi_{j}=\sum_{i=0}^{M} \pi_{i} \cdot P_{i j}, \quad j=0,1, \cdots, M \\
\sum_{i=0}^{M} \pi_{i}=1 .
\end{array}\right.
$$

The system throughput, denoted by $S(n)$, in packets per slot, conditioned on $N_{b}^{t}=n$, is given by

$S(n)=\sum_{l=0}^{M-n} \sum_{k=0}^{n} P^{c s}(l+k) \cdot B\left(M-n, l, q_{0}\right) \cdot B\left(n, k, q_{r}\right)$.

The steady-state throughput is then

$$
\bar{S}=\sum_{n=0}^{M} S(n) \cdot \pi_{n}
$$

Similarly, the expected channel backlog $\bar{N}_{b}$ is

$$
\bar{N}_{b}=\sum_{n=0}^{M} n \cdot \pi_{n}
$$

Thus, by Little's result [21], the average system delay is given by

$$
\bar{D}=\bar{N}_{b} / \bar{S}+1
$$

We also define the conditional offered traffic rate $G(n)$ [22] in packets per slot when the system is in state $n$, i.e.,

$$
G(n) \equiv(M-n) \cdot q_{0}+n \cdot q_{r}
$$

The average offered traffic rate $\bar{G}$ is given by

$$
\bar{G}=\sum_{n=0}^{M} G(n) \cdot \pi_{n}
$$

3) Conditional Success Probability $P^{s}(i)$ : Define $A_{j \mid i}$ and $S_{k}(i)$ as follows:

$$
\begin{aligned}
& A_{j \mid i}\left.\equiv \begin{array}{l}
\text { event that bit } j \text { of the captured } \\
\text { packet is successfully received } \\
\text { given } i \text { other interfering packets } \\
\text { transmit in the same time slot }
\end{array}\right\} \\
& j=1,2, \cdots, N ; \quad i=0,1, \cdots, M-1 \\
& S_{k}(i) \equiv \sum_{j_{1}<j_{2}<\cdots<j_{k}} \operatorname{Prob}\left\{A_{j_{1} \mid i} \cap A_{j_{2} \mid i} \cap \cdots \cap A_{j_{k} \mid i}\right\} \\
& S_{1}(i) \equiv \sum_{j} \operatorname{Prob}\left\{A_{j \mid i}\right\} \\
& S_{2}(i) \equiv \sum_{j_{1}<j_{2}} \operatorname{Prob}\left\{A_{j_{1} \mid i} \cap A_{j_{2} \mid i}\right\}
\end{aligned}
$$

Although the number of other interfering packets in a time slot is a random variable, it remains constant during the reception of the tagged packet. ${ }^{1}$ Therefore,

$$
\begin{aligned}
\operatorname{Prob}\left\{A_{j_{1} \mid i} \cap A_{j_{2} \mid i} \cap \cdots \cap A_{j_{k} \mid i}\right\} \\
=\operatorname{Prob}\left\{A_{j_{1}^{*} \mid i} \cap A_{j_{2}^{*} \mid i} \cap \cdots \cap A_{j_{k}^{*} \mid i}\right\} \\
=\operatorname{Prob}\left\{\begin{array}{l}
k \text { arbitrary bits of the tagged } \\
\text { packet are successfully decoded } \\
\text { given } i \text { other interfering packets } \\
\text { transmit in the same time slot }
\end{array}\right\} \\
\forall j_{1}<j_{2}<\cdots<j_{k} ; \quad i=0,1, \cdots, M-1
\end{aligned}
$$

where $\left\{j_{1}^{*}, j_{2}^{*}, \cdots, j_{k}^{*}\right\}$ is an arbitrarily selected set.

$S_{k}(i)$ is summed over all $\left(\begin{array}{l}N \\ k\end{array}\right)$ choices of $k$ events out of the available events $A_{1 \mid i}, A_{2 \mid i}, \cdots, A_{N \mid i}$. Define

$$
P(k \mid i) \equiv \operatorname{Prob}\left(A_{j_{1}^{*} \mid i} \cap A_{j_{2}^{*} \mid i} \cap \cdots \cap A_{j_{k}^{*} \mid i}\right) .
$$

Then, we obtain

$$
\begin{aligned}
S_{k}(i) & =\left(\begin{array}{c}
N \\
k
\end{array}\right) \operatorname{Prob}\left\{A_{j_{1}^{*} \mid i} \cap A_{j_{2}^{*} \mid i} \cap \cdots \cap A_{j_{k}^{*} \mid i}\right\} \\
& =\left(\begin{array}{c}
N \\
k
\end{array}\right) P(k \mid i) \\
& =\left(\begin{array}{c}
N \\
k
\end{array}\right) \sum_{j=0}^{k} P(k, j \mid i) \\
& =\left(\begin{array}{c}
N \\
k
\end{array}\right) \sum_{j=0}^{k} P(k \mid j, i) \cdot P(j \mid i)
\end{aligned}
$$

where

$$
P(k, j \mid i) \equiv \operatorname{Prob}\left\{\begin{array}{l}
k \text { bits of the tagged packet are } \\
\text { successfully decoded, and } j \text { bits } \\
\text { among these } k \text { bits are " } 1 \text { " bit } \\
\text { given } i \text { other interfering packets } \\
\text { transmit in the same time slot }
\end{array}\right\} .
$$

Since the number of " 1 " bits among $k$ bits, i.e., $j$, is independent of the number of other interfering packets, i.e., $i$, and we assume that " 1 " bit and " 0 " bit occur with equal probability, then we have

$$
P(j \mid i)=P(j)=B\left(k, j, \frac{1}{2} .\right)
$$

The term $P(k \mid j, i)$ can be derived as

$$
P(k \mid j, i)=\sum_{k_{1}=0}^{i}\left[\sum_{k_{2}=0}^{T h-1} B\left(k_{1}, k_{2}, \frac{1}{2}\right)\right]^{k-j} \cdot B\left(i, k_{1}, P_{v}\right)
$$

where $P_{v}=(d(d-1)) / c-1$.

Assume that we use random error-correcting codes (e.g., $\mathrm{BCH}$ codes) [23], and the codes can correct $t$ or fewer errors among $N$ bits. Then, a packet is successfully received only if there are at least $N-t$ correct bits in a received packet.

Define

$$
Q^{s}(k \mid i) \equiv \operatorname{Prob}\left\{\begin{array}{l}
\text { at least } k \text { bits among } N \text { bits } \\
\text { of tagged packet are correct } \\
\text { given } i \text { other interfering packets }
\end{array}\right\} .
$$

\footnotetext{
${ }^{1}$ The tagged packet is the captured packet.
} 
We can obtain [24]

$$
\begin{aligned}
Q^{s}(k \mid i)= & S_{k}(i)-\left(\begin{array}{c}
k \\
k-1
\end{array}\right) S_{k+1}(i)+\cdots \\
& +(-1)^{N-k}\left(\begin{array}{c}
N-1 \\
k-1
\end{array}\right) S_{N}(i) .
\end{aligned}
$$

Therefore, the conditional success probability is given by

$$
P^{s}(i)=Q^{s}(N-t \mid i-1)
$$

where

$$
\begin{aligned}
S_{k}(i)= & \left(\begin{array}{l}
N \\
k
\end{array}\right) \sum_{j=0}^{k} \sum_{k_{1}=0}^{i}\left[\sum_{k_{2}=0}^{T h-1} B\left(k_{1}, k_{2}, \frac{1}{2}\right)\right]^{k-j} \\
& \cdot B\left(i, k_{1}, P_{v}\right) \cdot B\left(k, j, \frac{1}{2}\right) .
\end{aligned}
$$

4) Optical Hard Limiter: In order to improve the performance, the interference power intensity must be reduced. One way to reduce the interference is to place an optical hard limiter [8], [10], [25] before the correlator. If an input light intensity $x$ to an ideal optical hard limiter is greater than or equal to 1 , then the output is clamped to 1 ; otherwise, it is 0 . The ideal optical hard limiter can improve the performance by excluding some combination of interference patterns which cause errors. An example of the effect of the optical hard limiter is shown in Fig. 3. In this figure, we consider an arbitrary bit $\alpha$. Without the optical hard limiter, the output level is 5 , which is above the threshold 3 of the threshold detector, but with the optical hard limiter, the output level is only 2 , which is below the threshold.

The analysis with the optical hard limiter follows the same procedure as in Section III-A2. We only have to derive the conditional success probability $P^{s}(i)$. Since pulse $\beta$ is hit when other packets initiate at vulnerable positions of pulse $\beta$, we can obtain

$$
\begin{aligned}
S_{k}(i)= & \left(\begin{array}{c}
N \\
k
\end{array}\right) \sum_{j=0}^{k} \sum_{\xi=0}^{i} \sum_{k_{0}, \ldots, k_{d-1} \in I_{\xi}}\left(\begin{array}{c}
\xi \\
k_{0}, \cdots, k_{d-1}
\end{array}\right) \\
& \cdot \prod_{\beta \in I_{d}}\left(P_{h, \beta}\right)^{k_{\beta}} \cdot\left(1-\prod_{\beta \in I_{d}} P_{\beta}\right)^{k-j} \\
& \cdot B\left(i, \xi, P_{v}\right) \cdot B\left(k, j, \frac{1}{2}\right)
\end{aligned}
$$

where $I_{\xi}, I_{d}$, and $k_{\beta}$ are as shown in (20a) at the bottom of the page and $P_{v}=d(d-1) /(c-1), P_{h, \beta}=1 / d, P_{\beta}=1-\left(\frac{1}{2}\right)^{k_{\beta}}$, $\forall \beta \in I_{d}$. Therefore, the conditional success probability is

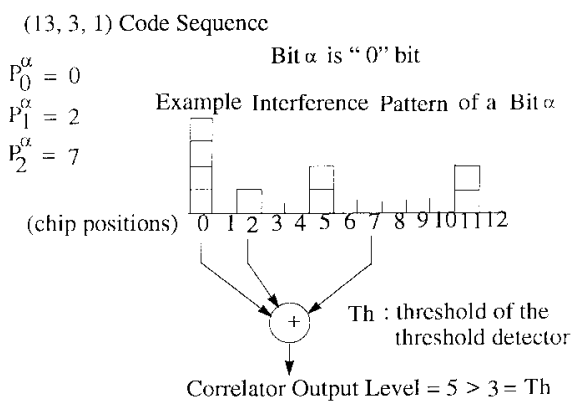

(a)

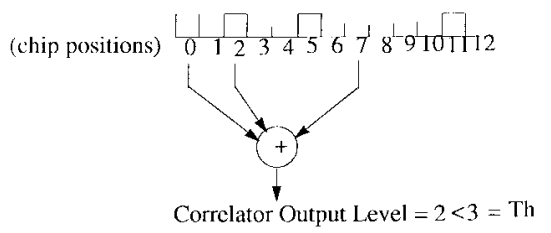

(b)

Fig. 3. Illustration of the effect of the optical hard limiter.

given by

$$
P^{s}(i)=Q^{s}(N-t \mid i-1)
$$

where $Q^{s}(k \mid i)$ is given by (17).

5) Numerical Example: In this subsection, we present some numerical results on the throughput and delay of centralized slotted TH/OOK CDMA packet network. Fig. 7 shows the delay versus throughput as a function of $c$ with parameters $M=20, N=127, d=3$, and $q_{r}=0.8$. The performance improves as the signature length $c$ is increased. However, the improvement is negligible when $c$ is very large because, in this case, the probability of successful transmission is dominated by the capture effect.

These figures also reveal the bistable behavior typical of the slotted ALOHA protocol [19], [26], i.e., the system possesses two statistically stable equilibrium points, one in a desirable low-delay region, and the other in an undesirable high-delay region. In Fig. 4, we plot the probability mass function (pmf) of the backlog size as a function of $q_{0}$ to illustrate the bistable mechanism. When $q_{0}=0.01$, the probability is concentrated on low backlog size, i.e., the system is stable; when $q_{0}=$ 0.019 , the pmf is bimodal, i.e., there are two stable equilibrium points; and when $q_{0}=0.03$, the pmf is skewed on high backlog size, and the system is saturated.

We can maximize the throughput by selecting $q_{r}$. In Fig. 5, we plot the delay versus throughput as a function of $q_{r}$; the envelope, ${ }^{2}$ which is not shown in this figure, of this group of curves is the delay versus maximum throughput curve. We

\footnotetext{
${ }^{2}$ The envelope of a group of curves is the curve which is tangential to all of these curves.
}

$$
\begin{aligned}
& I_{\xi} \equiv\left\{\begin{array}{l|l}
\left(k_{0}, \cdots, k_{\beta}, \cdots, k_{d-1}\right) & \begin{array}{l}
k_{0}+\cdots+k_{\beta}+\cdots+k_{d-1}=\xi \\
0 \leq k_{\beta} \leq \xi \\
\beta=0,1, \cdots, d-1
\end{array}
\end{array}\right\} \\
& I_{d} \equiv\{\beta: \beta=0,1, \cdots, d-1\} \\
& k_{\beta} \equiv \text { number of interfering packets hitting pulse } \beta
\end{aligned}
$$




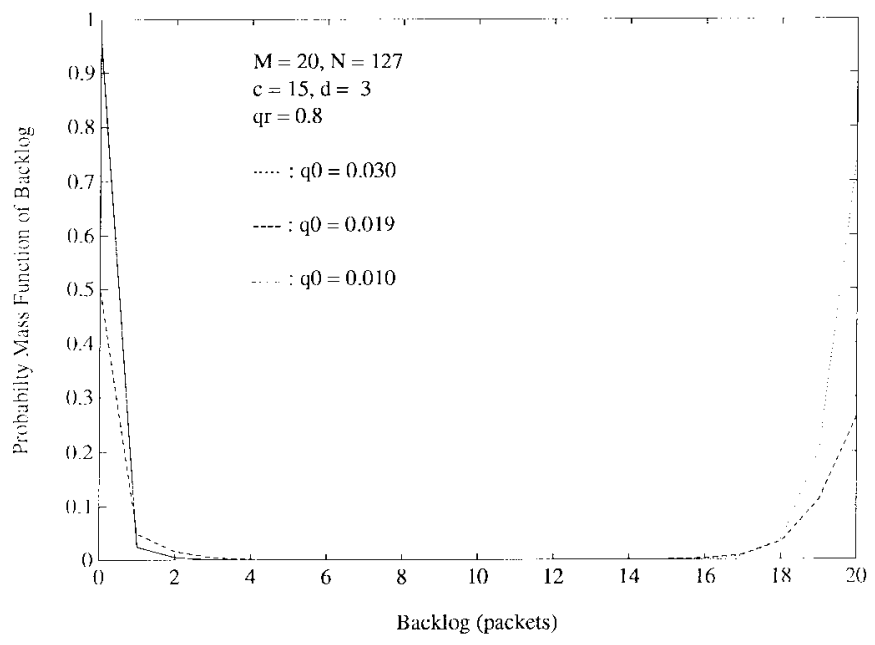

Fig. 4. Probability mass function of backlog size.

expect that the performance will improve if error-correcting code is employed. However, in Fig. 7, the effectiveness of random error-correcting codes (we use $\mathrm{BCH}$ codes [23]) is not as good as expected. This is due to the high correlation of probabilities of bit error in a packet. We also plot the normalized throughput ${ }^{3}$ in Fig. 6 for $\mathrm{BCH}$ codes with $t$-errorcorrecting capability. In our case, $N=127, t=2$ and 5, and the code rates are 0.8898 and 0.7244 , respectively [23]. When FEC is not added (i.e., $t=0$ ), the throughput is already very close to its maximum value which is obtained by the capture effect only. Furthermore, as is mentioned above, the random error-correcting codes are not as effective as expected in this system. Therefore, the normalized throughput decreases as $t$ increases. Next, we examine the effect of the optical hard limiter on the performance of the network. In Fig. 7, we find that dramatic performance improvement is obtained by employing an optical hard limiter.

\section{B. FH/TH/OOK Fiber-Optic CDMA Packet Networks}

1) System Model and Assumptions: In this section, we employ a hybrid scheme $\mathrm{FH} / \mathrm{TH} / \mathrm{OOK}^{4}$ which combines frequency-hopped (FH) CDMA and time-hopped (TH) CDMA. Each packet is encoded by a two-layered encoding process. The inner layer encoding, which is the same as $\mathrm{TH} / \mathrm{OOK}$, uses a $\mathrm{TH}$ signature pattern (inner signature) to encode bits. FH signature patterns (outer signature) are used in the outer layer encoding process. We assume that $q$ frequency slots can be chosen. The optical signal from a transmitter is hopped from slot to slot by changing the frequency at certain instants in time called hop epochs. The intervals of time between two consecutive hop epochs are called hop intervals. We consider only fixed-rate hopping, so all intervals are of the same length $T_{\text {hop }}$. Furthermore, we assume that the hop interval $T_{\text {hop }}$ is equal to the bit interval $T_{b}$.

We make the same assumptions as in Section II-A. Besides, we assume that random frequency-hopping patterns are used. If a user wants to transmit a packet, then in each hop interval, the user randomly and independently chooses one of the $q$

${ }^{3}$ Normalized throughput $=$ code rate $\cdot$ throughput.

${ }^{4}$ Some prefer different names, e.g., multiwavelength, multicolor CDMA.

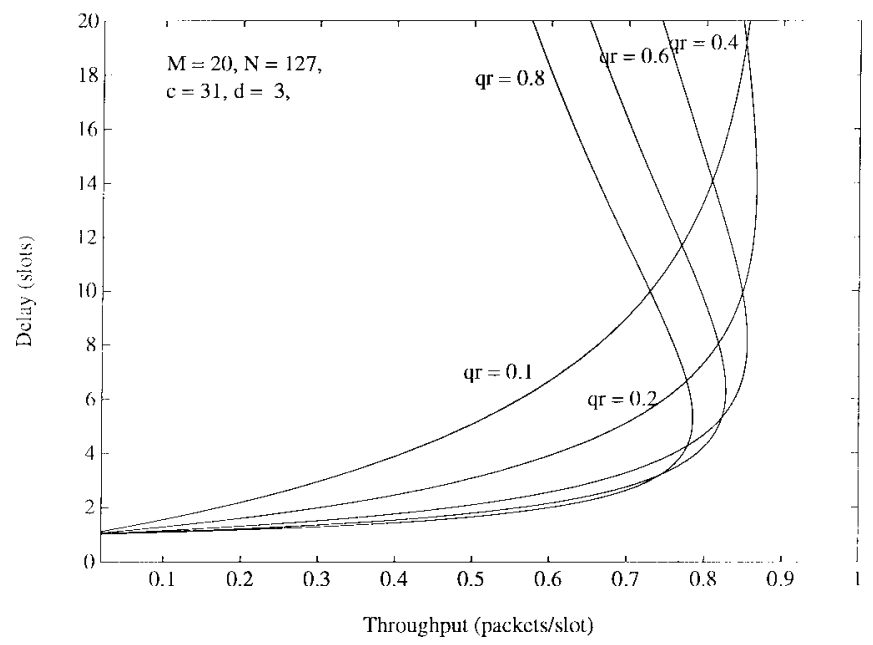

Fig. 5. Delay versus throughput as a function of $q_{r}$.

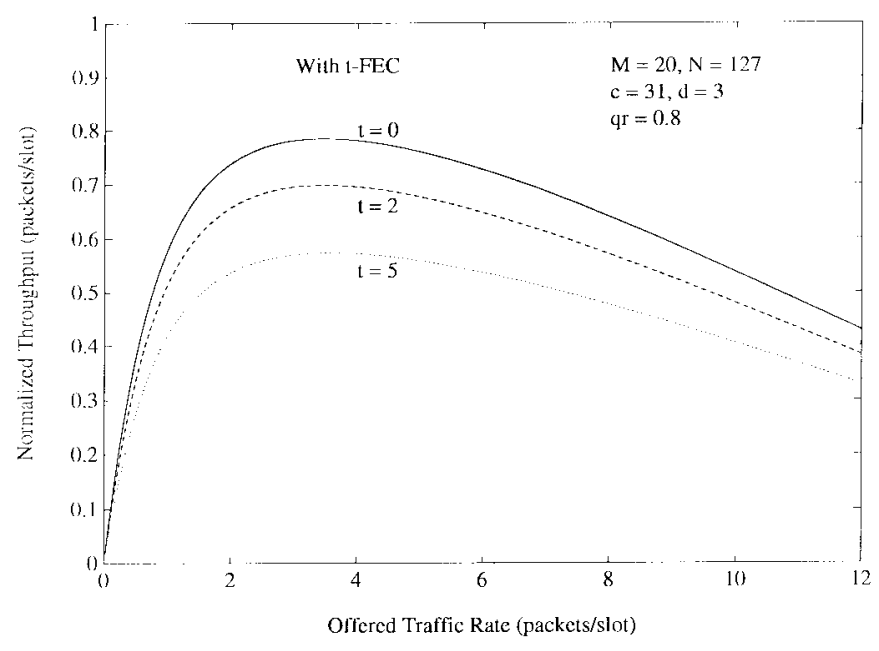

Fig. 6. Normalized throughput versus offered traffic rate with $t$-FEC.

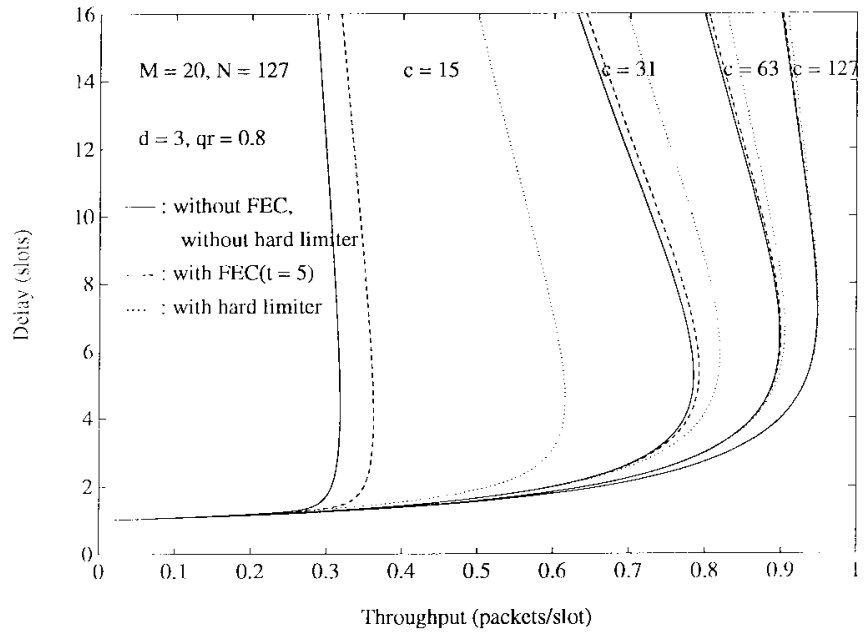

Fig. 7. Delay versus throughput for centralized slotted TH/OOK CDMA.

available frequency slots to transmit. We also employ random error correcting codes or an optical hard limiter to enhance the performance.

2) Analysis: We follow the same procedure as in the previous sections to derive throughput and delay performance. The only change required is the conditional success probability 
$P^{s}(i)$ in Section III-A2, namely, the probability that the captured packet is successfully received given that $i$ packets transmit in the same time slot. We obtain

$$
\begin{aligned}
S_{k}(i)= & \left(\begin{array}{l}
N \\
k
\end{array}\right) \sum_{j=0}^{k} \sum_{k_{1}=0}^{i}\left[\sum_{k_{2}=0}^{T h-1} B\left(k_{1}, k_{2}, \frac{1}{2 q}\right)\right]^{k-j} \\
& \cdot B\left(i, k_{1}, P_{v}\right) \cdot B\left(k, j, \frac{1}{2}\right)
\end{aligned}
$$

and

$$
P^{s}(i)=Q^{s}(N-t \mid i-1)
$$

where

$$
\begin{aligned}
Q^{s}(k \mid i)= & S_{k}(i)-\left(\begin{array}{c}
k \\
k-1
\end{array}\right) S_{k+1}(i)+\cdots \\
& +(-1)^{N-k}\left(\begin{array}{c}
N-1 \\
k-1
\end{array}\right) S_{N}(i) .
\end{aligned}
$$

Similarly, when an optical hard limiter is used in the network, we can obtain the same form of $S_{k}(i)$ as in (20), with only minor modification

$$
P_{\beta}=1-\left(1-\frac{1}{2 q}\right)^{k_{\beta}}
$$

and

$$
P^{s}(i)=Q^{s}(N-t \mid i-1) .
$$

3) Numerical Example: In this subsection, we present some numerical results on the throughput and delay of a centralized slotted FH/TH/OOK CDMA network.

In Fig. 8, we plot the delay versus throughput as a function of $q$, with parameters $M=20, N=127, c=15, d=3$, and $q_{r}=0.8$. As $q$ increases, the performance improves, but for $q$ greater than 10, no further improvements can be made. When $t$-FEC or an optical hard limiter is employed, the performance also improves. In Fig. 8, for $q$ greater than 10, the curves merge to the curve for $q \rightarrow \infty$.

\section{Distributed Slotted Fiber-Optic CDMA PACKET NETWORKS}

\section{A. TH/OOK CDMA Packet Networks}

1) System Model and Assumptions: In this section, we consider the distributed slotted fiber-optic CDMA packet networks, and we use the same assumptions as in Section III. Besides, we assume that a transmitter has no dedicated receiver (i.e., nonpaired off) to communicate with; it can always find an idle receiver to receive its packets [14]. This results in an optimistic system performance. We assume a homogeneous network in which each node generates the same rate of traffic, and the destination is uniformly distributed among all nodes except the transmitting node. A packet is successfully received by a receiver if

1) the packet is captured by a receiver, i.e., there are no other packets initiated at the same chip position as the "tagged" packet;

2) the other interfering packets do not have sufficient signal strength to overwhelm the interference rejection capability of the receiver which captures the packet.

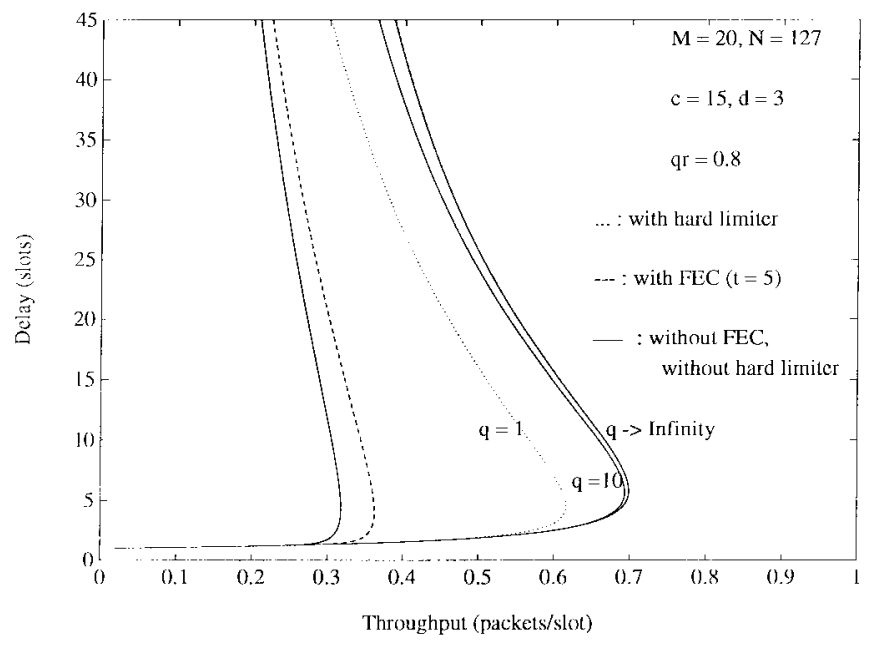

Fig. 8. Delay versus throughput for centralized slotted FH/TH/OOK CDMA.

Random error-correcting codes or an optical hard limiter are employed in the network.

2) Analysis: We consider a network with $M$ users, and we assume that the thinking users and blocked users transmit their packets in a slot with the same probability $p$ (i.e., $q_{0}=q_{r}=p$ ). Then the arrival process $I$ of the network is a binomial distribution with probability mass function

$$
f_{I}(i)= \begin{cases}\left(\begin{array}{c}
M \\
i
\end{array}\right) p^{i}(1-p)^{M-i}, & \text { if } i \leq M \\
0, & \text { if } i>M .\end{cases}
$$

We can define offered traffic rate to be

$$
G \equiv(M-n) \cdot q_{0}+n \cdot q_{r}=M \cdot p
$$

where $n$ is the backlog size, and with $q_{0}=q_{r}=p$, we have

$$
f_{I}(i)= \begin{cases}\left(\begin{array}{c}
M \\
i
\end{array}\right)\left(\frac{G}{M}\right)^{i}\left(1-\frac{G}{M}\right)^{M-i}, & \text { if } i \leq M \\
0, & \text { if } i>M .\end{cases}
$$

Let $P^{c s}(i)$ be the conditional packet success probability given that $i$ packets transmit in the same time slot. The conditional mean of the number of successful packets $K$ is given by $\boldsymbol{E}\{K \mid i\}=P^{c s}(i) \cdot i$. The steady-state throughput $S$ is the expected number of successful packets per slot, and

$$
\begin{aligned}
S & =\boldsymbol{E}\{K\}=\boldsymbol{E}\{\boldsymbol{E}\{K \mid I\}\} \\
& =\sum_{i=1}^{\infty} i \cdot P^{c s}(i) \cdot f_{I}(i) \\
& =\sum_{i=1}^{M} i \cdot\left(\begin{array}{c}
M \\
i
\end{array}\right)\left(\frac{G}{M}\right)^{i}\left(1-\frac{G}{M}\right)^{M-i} \cdot P^{c s}(i)
\end{aligned}
$$

where

$$
P^{c s}(i)=P^{c}(i) \cdot P^{s}(i)
$$

and

$$
P^{c}(i)= \begin{cases}1, & \text { if } i=1 \\ \left(1-\frac{1}{c}\right)^{i-1}, & \text { if } i \geq 2 \\ 0, & \text { otherwise }\end{cases}
$$




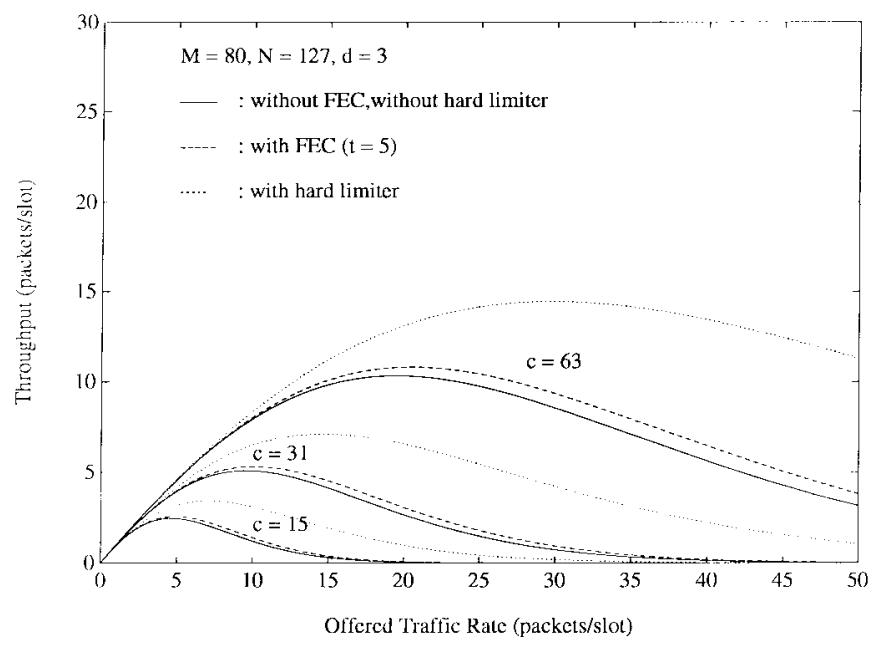

Fig. 9. Throughput versus offered traffic rate for distributed slotted TH/OOK CDMA.

and $P^{s}(i)$ is given by (18). Similarly, when the optical hard limiter is added, the analysis is the same as above, except that $P^{s}(i)$ is given by (21).

3) Numerical Example: In this subsection, we present some numerical results on the throughput of a distributed slotted TH/OOK CDMA network. In Fig. 9, we plot the throughput versus offered traffic rate as a function of $c$, with parameters $M=80, N=127$, and $d=3$. In a distributed network, the number of successful transmissions in a slot can be more than one. As before, the throughput increases as signature length $c$ increases. However, as $c$ increases, the throughput increases very rapidly, and does not saturate as in the centralized network. In Fig. 9, we plot the throughput versus offered traffic rate when $t$-FEC is added with $t=5$. Again, the improvement is very limited due to the high correlation between probabilities of bit error among bits in the tagged packet. When the optical hard limiter is employed, the improvement is dramatic. Under the same offered traffic rate, the probability of successful transmission for a packet in distributed networks will be higher than that in centralized networks. Therefore, the average delay of a packet to be successfully received will be smaller in distributed networks.

\section{B. FH/TH/OOK CDMA Packet Networks}

1) System Model and Analysis: In this section, we consider a distributed slotted network employing the FH/TH/OOK scheme described in Section III-B. The analysis is the same as in the previous section. Throughput $S$ is given by

$$
S=\sum_{i=1}^{\infty} i \cdot P^{c s}(i) \cdot f_{I}(i)
$$

where $f_{I}(i)$ is given by (28), $P^{c}(i)$ is given by (30), and $P^{s}(i)$ 's are given by (23) and (26) without an optical hard limiter and with an optical hard limiter, respectively.

2) Numerical Example: In this subsection, we present some numerical results on the throughput of a distributed slotted FH/TH/OOK CDMA network. In Fig. 10, we plot the throughput versus offered traffic rate as a function of

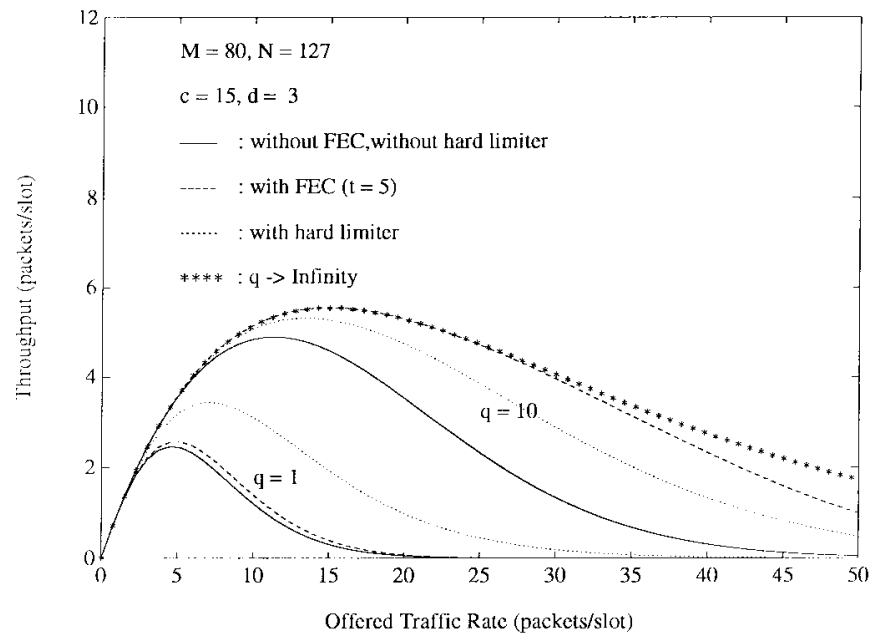

Fig. 10. Throughput versus offered traffic rate for distributed slotted FH/TH/OOK CDMA.

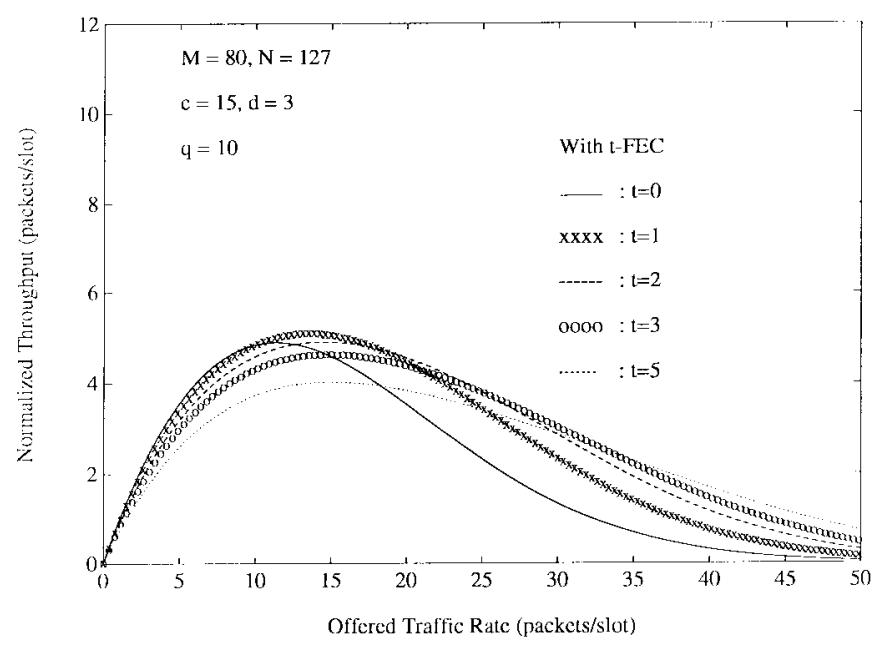

Fig. 11. Normalized throughput versus offered traffic rate with $t$-FEC.

$q$, with $M=80, N=127, c=15$, and $d=3$. The throughput increases as $q$ increases. When $q$ is greater than 40, the throughput increments are limited since the throughput is dominated by the capture effect. In Fig. 10, we plot the throughput versus offered traffic rate when $t$ FEC is employed with $t=5$. In this case, the throughput improvement by random error-correcting code is very obvious. This is because we employ frequency hopping to randomize the dependence characteristics among bits in a packet. In Fig. 10, the improvement on throughput by $t$-FEC is greater than that by an optical hard limiter because of the randomizing effect of the FH scheme that renders random error-correcting codes more effective. We also plot the normalized throughput for $t$-FEC codes in Fig. 11. There is an optimum value of the FEC code rate which achieves the maximum normalized throughput. In this case, the corresponding code rate is 0.9449 .

\section{CONCLUSIONS}

In this paper, we have analyzed the performance of centralized and distributed slotted TH/OOK and FH/TH/OOK CDMA packet networks. We extract some useful information, i.e., 
vulnerable positions and interference sets, from the signature patterns by the orthogonality properties.

In each scheme, we show that the performance can be improved by adding $t$-FEC codes or an optical hard limiter. In $\mathrm{TH} / \mathrm{OOK}$, random error correcting codes are not as effective as an optical hard limiter because of the correlation among bits in a packet. However, in $\mathrm{FH} / \mathrm{TH} / \mathrm{OOK}$, the correlation among bits is reduced by the $\mathrm{FH}$ randomizing effect, and the performance improvement by random error-correcting codes is greater than that by an optical hard limiter.

The numerical results indicate that the required throughput and/or delay of fiber-optic CDMA networks can be achieved by properly selecting the code length $c$ of the signature and/or number of frequency slots $q$ (in the hybrid scheme). These two parameters $c$ and $q$ correspond to the processing gains in the conventional CDMA network. The system performance can be improved, or equivalently, the interference rejection capability of the CDMA networks can be enhanced by increasing the processing gains. Therefore, in some bursty and real-time networks with large number of users, CDMA is an appropriate choice.

\section{REFERENCES}

[1] K. P. Jackson et al., "Optical fiber delay-line signal processing," IEEE Trans. Microwave Theory Tech., vol. MTT-33, pp. 193-210, Mar. 1985.

[2] B. Moslehi, J. W. Goodman, M. Tur, and H. J. Shaw, "Fiber-optic lattice signal processing," Proc. IEEE, pp. 909-930, July 1984.

[3] J. K. Holmes, Coherent Spread Spectrum Systems. New York: WileyInterscience, Inc., 1982.

[4] C. L. Weber, G. K. Huth, and B. H. Batson, "Performance considerations of code division multiple-access systems," IEEE Trans. Veh. Technol., vol. VT-30, pp. 3-10, Feb. 1981.

[5] F. R. K. Chung, J. A. Salehi, and V. K. Wei, "Optical orthogonal codes: Design, analysis and applications," IEEE Trans. Inform. Theory, vol. 35, pp. 595-604, May 1989.

[6] H. Chung and P. V. Kumar, "Optical orthogonal codes-New bounds and an optimal construction," IEEE Trans. Inform. Theory, vol. 36, pp. 866-873, July 1990.

[7] F. Khansefid, H. Taylor, and R. Gagliardi, "Design of $(0,1)$ sequence sets for pulse coded systems," Univ. Southern California, Tech. Rep. CSI-88-03-03, Mar. 1988.

[8] S. S. Matsunaga, "On the performance of code division multiple access optical fiber communications," Univ. Southern California, May 1989.

[9] J. A. Salehi, "Code division multiple-access techniques in optical fiber networks-Part I: Fundamental principles," IEEE Trans. Commun., vol. 37, pp. 824-833, Aug. 1989.

[10] J. A. Salehi and C. A. Brackett, "Code division multiple-access techniques in optical fiber networks-Part II: Systems performance analysis," IEEE Trans. Commun., vol. 37, pp. 834-842, Aug. 1989.

[11] R. M. Gagliardi and S. Karp, Optical Communications. New York: Wiley, 1975.

[12] A. D. Gelman and D. L. Schilling, "A fiber optic CDMA network for real-time communication," in IEEE INFOCOM, pp. 1B.4.1-1B.4.8, 1988.

[13] J. Y. Hui, "Throughput analysis for code division multiple accessing of the spread spectrum channel," IEEE J. Select. Areas Commun., vol. SAC-2, pp. 482-486, July 1984.

[14] D. Raychaudhuri, "Performance analysis of random access packetswitched code division multiple access systems," IEEE Trans. Commun., vol. COM-29, pp. 895-901, June 1981.

[15] D. H. Davis and S. A. Gronemeyer, "Performance of slotted ALOHA random access with delay capture and randomized time of arrival," IEEE Trans. Commun., vol. COM-28, pp. 703-710, May 1980.

[16] S.-L. Su, "Performance analysis of spread spectrum networks," Ph.D dissertation, Univ. Southern California, Los Angeles, Dec. 1985.
[17] N. Abramson, "The throughput of packet broadcasting channels," IEEE Trans. Commun., vol. COM-25, pp. 117-128, Jan. 1977.

[18] L. Kleinrock and S. S. Lam, "Packet switching in a multiaccess broadcast channel: Performance evaluation," IEEE Trans. Commun., vol. COM-23, pp. 410-423, Apr. 1975.

[19] A. B. Carleial and M. E. Hellman, "Bistable behavior of ALOHA-type systems," IEEE Trans. Commun., vol. COM-23, pp. 401-410, Apr. 1975.

[20] L. Kleinrock, Queueing Systems, Vol. I: Theory. New York: WileyInterscience, 1975.

[21] D. Gross and C. M. Harris, Fundamentals of Queueing Theory, 2nd ed. New York: Wiley, 1985.

[22] D. Bertsekas and R. Gallager, Data Networks, 2nd ed. Englewood Cliffs, NJ: Prentice-Hall, 1992.

[23] G. C. Clark, Jr. and J. B. Cain, Error-Correcting Coding for Digital Communications. New York: Plenum, 1981.

[24] W. Feller, An Introduction to Probability Theory and Its Applications, Vol. I. New York: Wiley, 1968.

[25] A. A. Sawchuk and T. C. Strand, "Digital optical computing," Proc. IEEE, vol. pp. 758-779, July 1984.

[26] S. Tasaka, Performance Analysis of Multiple Access Protocols. Cambridge, MA: MIT Press, 1986

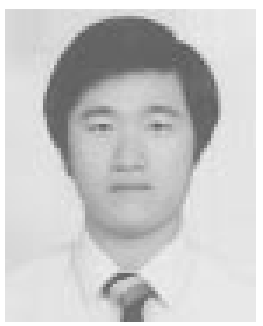

Cherng-Shung Hsu (S'88-M'92) was born in Taiwan, R.O.C., in 1956. He received the B.S. degree in telecommunications from National Chiao-Tung University, Hsinchu, Taiwan, in 1979, the M.S. degree in electrical engineering from National Taiwan University, Taipei, in 1981, and the Ph.D. degree in electrical engineering from the University of Southern California (USC), Los Angeles, in 1992. From 1982 to 1988, he was with the Chung-Shan Institute of Science and Technology (CSIST), LungTan, Taiwan, where he was an Assistant Researcher. He has been with CSIST as an Associate Researcher since 1992. His research interests include high speed communication networks and performance analysis, communication theory and systems, and personal communication networks.

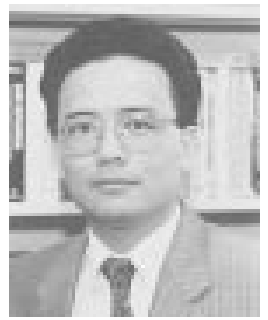

Victor O. K. Li (S'80-M'81-SM'86-F'92) was born in Hong Kong in 1954. He received the S.B., S.M., and Sc.D. degrees in electrical engineering and computer science from the Massachusetts Institute of Technology, Cambridge, MA, in 1977, 1979, and 1981, respectively.

Since February 1981, he has been with the University of Southern California (USC), Los Angeles, where he is Professor of Electrical Engineering and Director of the USC Communication Sciences Institute. His research interests include high speed communication networks, personal communication networks, distributed multimedia systems, distributed databases, queueing theory, graph theory, and applied probability. He has lectured and consulted extensively around the world.

Dr. Li chaired the Computer Communications Technical Committee of the IEEE Communications Society from 1987 to 1989, and the Los Angeles Chapter of the IEEE Information Theory Group from 1983 to 1985 . He chaired the Steering Committee of the International Conference on Computer Communications and Networks $\left(I C^{3} N\right)$ from 1992 to 1997, and was General Chair of the 1st Annual $I C^{3} N$, June 1992, Technical Program Chair of the Institution of Electrical Engineers (IEE) Personal Communication Services Symposium, June 1995, and Chair of the 4th IEEE Workshop on Computer Communications, October 1989. A member of ACM, Dr. Li has served as an editor of IEEE Network and of Telecommunication Systems, guest editor of IEEE JSAC and of Computer Networks and ISDN Systems, and is now serving as an editor of ACM Wireless Networks. He serves on the International Advisory Board of IEEE TENCON'90, IEEE TENCON'94, IEEE SICON'91, IEEE SICON'93, IEEE SICON/ICIE'95, the International Conference on Microwaves and Communications '92, and the International Symposium on Communications ' 91 . 